\title{
Special issue: Glycobiology on stem cells —editorial
}

\author{
Toshisuke Kawasaki $^{1}$ (D) Robert K. Yu ${ }^{2}$
}

Published online: 6 November 2017

(C) Springer Science+Business Media, LLC 2017

Stem cells differ from other kinds of cells in the body in several aspects. First, they can divide and renew themselves (self-renewing) for a long period of time, and second, they retain the ability to give rise to all cell types of the body (pluripotency). Stem cells are divided into two classes: embryonic stem cells and non-embryonic somatic or adult stem cells. Embryonic stem cells were first established from early mouse embryos in 1981 (Evans and Kaufman) and a convenient method to derive stem cells from human embryos was then developed in 1998 (Thomson). In 2006, a major breakthrough was achieved (Yamanaka) by identifying conditions that allow some adult cells to be "reprogrammed" genetically to a stem cell-like state. This new type of stem cell is called induced pluripotent stem (iPS) cells. Adult stem cells can be found among differentiated cells in a variety of tissues and organs. The bone marrow contains two kinds of stem cells: hematopoietic stem cells, which can form all the types of blood cells, and bone marrow stromal stem cells (also called mesenchymal stem cells), which can generate bone, cartilage and fat cells. The adult brain contains stem cells that are capable of generating the brain's three major cell types: neurons, astrocytes, and oligodendrocytes.

When stem cells give rise to specialized cells in a process called differentiation, the signals inside and outside

Toshisuke Kawasaki

tkawasak@fc.ritsumei.ac.jp

1 Research Center for Glycobiotechnology, Ritsumeikan University, Kusatsu, Shiga, Japan

2 Department of Neuroscience and Regenerative Medicine, Medical College of Georgia, Augusta University, Augusta, GA, USA cells trigger each step of the differentiation process. Glycans attached to proteins (glycoproteins) and sphingolipids (glycosphingolipids) are mainly located at the outermost surface of the cell. Significant alterations in the cellular glycoform may occur during differentiation and glycans could serve as specific stem cell markers and cellular differentiation biomarkers. In the last decade, many interesting findings have been reported in the field of glycobiology on stem cells. In addition, human iPS cells are already used as tools for drug development and disease modeling, and their most important potential application is the generation of cells and tissues that can be used for regenerative medicine. Stem cell research is obviously one of the most fascinating and exciting areas of contemporary biology including glycobiology.

This special issue comprises of 10 excellent review articles covering from early discoveries in stem cell research to the cutting edge of the present technologies. It embraces glycomics (glycoproteins, glycolipids and proteoglycans), signal transduction, regulation and metabolism, and three attractive original articles on human iPS cells and mesenchymal stem cells. We sincerely hope that this issue will contribute to a better understanding the crucial roles of glycans in stem cell research and stimulate further research in this evolving area. 